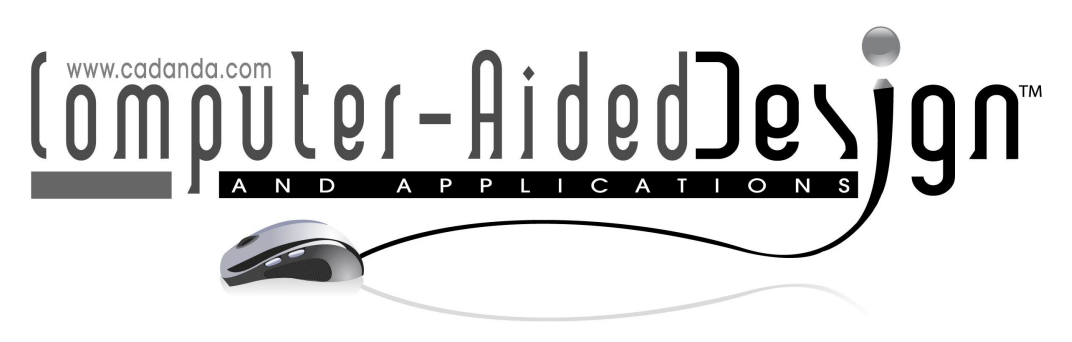

\title{
Algebraic Filtering of Surfaces from 3D Medical Images with Julia
}

\author{
Miroslav Jirik¹,3 (D), Antonio DiCarlo (D) , Václav Liska ${ }^{1,4}$ (D) and Alberto Paoluzzi² (D), \\ ${ }^{1}$ Biomedical Center, Faculty of Medicine in Pilsen, Charles University, Pilsen, Czech Republic, \\ jirik@lfp.cuni.cz \\ ${ }^{2}$ Roma Tre University, Department of Mathematics and Physics, Rome, Italy, \\ ${ }^{3}$ NTIS, Faculty of Applied Sciences, University of West Bohemia, Pilsen, Czech Republic, \\ ${ }^{4}$ Department of Surgery, University Hospital and Faculty of Medicine in Pilsen, Charles University, Pilsen, \\ Czech Republic,
}

Corresponding author: Miroslav Jirik, jirik@lfp.cuni.cz

\begin{abstract}
In this paper we introduce a novel algebraic filter, based on algebraic topology methods, to extract and smooth the boundary surface of any subset of voxels arising from the segmentation of a 3D medical image. The input of the Linear Algebraic Representation (LAR) Surface extraction filter (LAR-SURF) is defined as a chain, i.e., an element of a linear space of chains here subsets of voxels represented in coordinates as a sparse binary vector. The output is produced by a linear mapping between spaces of 3- and 2-chains, given by the boundary operator $\partial_{3}: C_{3} \rightarrow C_{2}$. The only data structures used in this approach are sparse arrays with one or two indices, i.e., sparse vectors and sparse matrices. This work is based on LAR algebraic methods and is implemented in Julia language, natively supporting parallel computing on hybrid hardware architectures.
\end{abstract}

Keywords: Medical 3D, Computational Topology, Linear Algebraic Representation, LAR, Julia, Surface Extraction

DOI: https://doi.org/10.14733/cadaps.2021.468-485

\section{Introduction}

Isosurface extraction to produce geometric models of surfaces from volumetric data is important in many applications. It is often used for interactive visualization of medical data and/or for flow modeling [21].

The most popular algorithm used for surface extraction from volume images is the Marching Cubes (MC). The algorithm was described by Lorentsen and Cline [13] in 1987. A survey of MC-inspired algorithms was published in 2006 [15]. The algorithm is based on considering the small cubes defining the volumetric image. Each corner vertex of each cube is associated with input volumetric data, typically average of the incident voxel data. MC traverses the data cube-by-cube, and constructs a triangulated iso-surface by using a lookup 
table depending on pattern of transitions between values of adjacent cube vertices. The main disadvantages of this method are time requirements, ambiguity, and hole generation. Some of them were discovered shortly after the algorithm was introduced. In 1991 Nielson and Hamman described an Asymptotic Decider to solve the ambiguity problem on the faces of the cube. Natarajan noted that the ambiguity problem also occurs with uniform samples [14]. In 1995 Chernyaev extended the number of lookup cases to 33 [5]. More recently, the algorithm was updated by Custodio, Pesco, and Silva to enhance the quality of the iso-surface triangulation [7].

Several alternative methods have been developed, including a method for surface extraction from a grid of field values using particle attraction; a system was described by Crossno and Angel in [6]. A graph processing that tracks the boundary cell-face adjacencies is described in [12]. Some parallel algorithms for iso-surface extraction are discussed in [1]. A data-parallel algorithm, implemented in OpenCL, that runs entirely on the GPU is presented in [23]. A Linear Algebraic Representation approach, parallelized using the OpenCL framework on Linux, was introduced in [16].

In the present paper we discuss a distributed approach for surface extraction, where the LAR-SURF (Linear Algebraic Representation Surface Extraction) filter is based on basic linear algebra and algebraic topology, using linear spaces $C_{p}$ of chains (of cells) of dimension $0 \leq p \leq 3$ and the boundary matrix $\left[\partial_{3}\right]: C_{3} \rightarrow C_{2}$.

Input volumetric data are represented by a $3 \mathrm{D}$ voxel array and can be generated, e.g., by segmentation of a computed tomography (left image in Fig. 4). A decomposition of input volumetric data into small submatrices called bricks is performed, then the binary coordinate vector of each segment (mathematically, a chain) of voxels is generated, and its boundary is computed by multiplying the boundary matrix. The resulting output is a sparse binary vector encoding the LAR representation of the boundary surface. This embarrassing parallel data decomposition is used to compute the boundary patches independently within each brick. All pathes are finally joined and smoothed via the Taubin algorithm [25].

The paper is organized as follows. Section 2 provides the basic topological and geometrical concepts needed to understand the LAR-SURF method, including the building of boundary matrices, the map from Cartesian indices to linear indices, and the Taubin smoothing method. Section 3 discusses the parametric design of the unit block filtered by the parallel algorithm, including the block decomposition, the sparsity rate of the sparse arrays used, and the block-level parallelism. Section 4 concerns to the algorithm implementation in Julia, and a discussion of the parallel workflow. Section 5 presents some examples of algorithm execution on a liver and its portal system. Section 7 shortly describes extensions of this approach, in particular the implementation with Julia's support for GPU parallelism and the multi-segmentation of medical images.

\section{Background}

Some basic concepts of solid modeling, and in particular the foundational idea of representation scheme, as well as a few basic concepts of algebraic topology, are shortly introduced in this section, including the computation of the matrix of a boundary operator between chain spaces.

\subsection{Representation Scheme}

A representation scheme for solid modeling is a mapping between a space of mathematical models and a space of symbolic representations, like those generated by a formal grammar. Solid pointsets (i.e., " $r$-sets") are defined in [20] as compact (bounded and closed) regular and semianalytic ${ }^{1}$ subsets of the $d$-space. A large number of representation schemes were defined in the past forty years, including the two main classes of (a) boundary representations (" $B$-reps"), where the solid model is represented through its boundary elements, i.e. faces, edges and vertices, and (b) decompositive/enumerative representations [20], that are a

\footnotetext{
${ }^{1}$ Semianalytic sets, studied in algebraic geometry, they are solutions of systems of polynomials; are closed under (regularized) set union, intersection and difference, and therefore constitute a Boolean algebra [18].
} 
decomposition of either the object or the embedding space, respectively, into a well-defined cellular complex. In particular, a boundary representation provides a cellular decomposition of the object's boundary into cells of dimension zero (vertices), one (edges), and two (faces). Medical imaging can be classified as the enumerative representation of cellular decompositions of organs and tissues [16], in particular, as subsets of $3 D$ volume elements (voxels) from 3D medical image.

\subsection{Linear Algebraic Representation}

The Linear Algebraic Representation (LAR), introduced in [9], aims at representing the chain complex [8, 17] generated by a piecewise-linear geometric complex embedded either in 2D or in 3D. This representation provides a minimal characterization of geometry and topology of a cellular complex, through (a) the embedding mapping $\mu: C_{0} \rightarrow \mathbb{E}^{d}$ of 0 -cells (vertices), and (b) a description of $d$-cells and/or $(d-1)$-cells as subsets of vertices. When evaluated, it is able to return the whole chain complex:

$$
C \bullet=\left(C_{p}, \partial_{p}\right):=C_{3} \underset{\partial_{3}}{\stackrel{\delta_{2}}{\leftrightarrows}} C_{2} \underset{\partial_{2}}{\stackrel{\delta_{1}}{\leftrightarrows}} C_{1} \underset{\partial_{1}}{\stackrel{\delta_{0}}{\leftrightarrows}} C_{0} .
$$

i.e., the whole sequence of graded linear chain spaces $C_{p}$, and all linear boundary $\partial_{p}$ and coboundary $\delta_{p}$ maps, with $\delta_{p}=\partial_{p-1}^{\top}$. The domain of LAR is the set of chain complexes generated by cell $d$-complexes $(2 \leq d \leq 3)$. The computer representations of LAR are sparse binary matrices to represent both the operators and the bases of chain spaces. Note that in algebraic topology a $p$-chain is defined as a linear combination of $p$-cells with scalars from a field. When the scalar coefficients are from $\{-1,0,+1\}$, a chain may represent any (oriented) subset of cells from the cellular complex. Scalars from $\{0,1\}$ are used for non-oriented complexes.

We may, therefore, get the $(p-1)$-boundary $\partial_{p} c_{p}$ of any $p$-chain $c_{p}$, by multiplying of the coordinate representation $\left[\partial_{p}\right]$ of the boundary operator times the coordinate representation $\left[c_{p}\right]$ of the chain in terms of such scalars, i.e., by a matrix-vector product $\left[\partial_{p}\right]\left[c_{p}\right]$.

It is possible to show that the LAR representation scheme is very expressive, i.e., that it has a large domain, including collections of: line segments, quads, triangles, polygons, meshes; pixels, voxels, volume images; B-reps, enumerative and decompositive representations of solids. In this paper we apply LAR methods to computation of boundary representations of solid models from segmentation (labeling) of 3D medical images. To display a triangulation of boundary faces in their proper position in space, the information required is contained in the geometric chain complex (GCC):

$$
\left.\mu: C_{0} \rightarrow \mathbb{E}^{3},\left(\delta_{0}, \delta_{1}, \delta_{2}\right) \equiv \text { (geom, top }\right)=(\mathrm{V},(\mathrm{EV}, \mathrm{FE}, \mathrm{CF}))
$$

Note that ordered pairs of letters from V,E,F,C, correspond to Vertices $\rightarrow$ Edges $\rightarrow$ Faces $\rightarrow$ Cells into the Column $\rightarrow$ Row order of matrix maps of linear operators. The boundary 2-cycle surface (possibly non connected) is transformed into a standard B-rep [22] using the information store within a GCC. The geometry geom is given by the embedding matrix $\mathrm{V}$ of vertices (0-cells); the topology top by the three sparse matrices (EV, FE, $\mathrm{CF})$ of coboundaries $\left(\delta_{0}, \delta_{1}, \delta_{2}\right)$ of the chain complex describing the space arrangement [19].

\section{Construction of the Boundary Matrix $\partial_{d}$}

First, let us fix an ordering for the cells of a partition of the input data, i.e., the matrix of vertex coordinates $\mathrm{V}$, and the arrays of arrays of vertex indices specifying edges EV, pixels FV, and voxels CV, i.e., for each $0-$, 1-, $2-$, and 3-elements of a cell partition V,E, F, C of a 3D image. These orderings fix the $p$-bases for the linear spaces $C_{p}$ of $p$-chains $(0 \leq p \leq 3)$. The matrix $M_{p}=\left(m_{i, j}\right)$ is called the characteristic matrix of the $p$-basis, where each $p$-cell is expressed as a subset of vertices ( 0 -cells), so that $m_{i, j}=1$ if and only if the $j$-th 0 -cell $c_{0}^{j}$ belongs to the boundary of $i$-th $p$-cell $m_{i, j}$, and $m_{i, j}=0$ otherwise. 


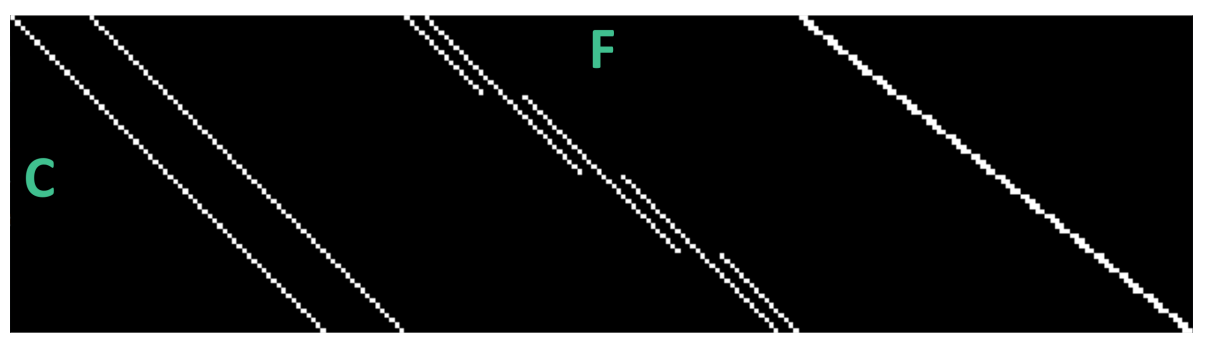

Figure 1: The binary image of the sparse coboundary matrix $\left[\delta_{2}\right]=\left[\partial_{3}\right]^{t}: C_{2} \rightarrow C_{3}$, built for a small volumetric data (or a brick) with shape $(4,4,4)$. Note that the number of rows equals the size $4 \times 4 \times 4=64$ of the voxel set; the number of columns is $d n(1+n)^{d-1}=3 \times 4 \times 25=300$. Of course, the number of non-zeros per row (cardinality of the facet set of a single voxel) is six, whereas the number of non-zeros per column is generally two, apart from boundary facets. The letter $\mathrm{F}$ stands for Faces, on matrix columns, and the $\mathrm{C}$ stands for Cells (3-cells) on matrix rows.

The computation of the boundary matrix $\left[\partial_{p}\right]$ begins by computing the compatible product of the two characteristic matrices $M_{p-1} M_{p}^{t}$. Let us note that the product of binary matrices is not binary, so that by computing the (sparse) matrix product $\left(M_{p-1} M_{p}^{t}\right)=\left(n_{i, j}\right)$, with $n_{i, j}=\sum_{k} m_{i, k} m_{k, j}$, we get for each $n_{i j}$ the number of vertices shared by $c_{p-1}^{i}$ and $c_{p}^{j}$. When this number equals the cardinality of $c_{p-1}^{i}$, the elementary chain is contained in the boundary of $c_{p}^{j}$.

In volume imaging data, comprised cubic 3-cells and square 2-cells between adjacent pairs of 3-cells, everywhere we get $n_{i, j}=4$, we may state $c_{2}^{i} \subset \partial c_{3}^{j}$. Therefore, in each $j$ column of $M_{2} M_{3}^{t}=\left(n_{i, j}\right)$, implementing the map FC: CV $\rightarrow \mathrm{VF}$, we have exactly six rows where $n_{i, j}=4$, since a cube (3-chain) has six boundary faces (a 2-chain) on its boundary. Of course, it will contain six non-zero elements for column. It may be worth remembering that every 3-cell (voxel) of the volumetric data has exactly six 2-faces.

Finally, consider the linear boundary operator $\partial_{p}: C_{p} \rightarrow C_{p-1}$. As such, it contains by columns the representation of domain basis elements, expressed as a linear combination of the basis elements of the range space. Therefore, the operator matrix $\left[\partial_{p}\right]$ is readily obtained by setting

$$
\left[\partial_{p}\right](i, j)=1 \quad \text { if } \quad n_{i, j}=\sum_{k} m_{i, k} m_{k, j}=\# c_{p-1}^{i} \quad \text { and } \quad\left[\partial_{p}\right](i, j)=0 \quad \text { otherwise. }
$$

To this purpose concider the rows of matrix $\left[\partial_{3}\right]^{t}$ in Figure 1, where the matrix is transposed for convenience. The unit incidence coefficients in $\left[\partial_{3}\right]$, shown as white pixels, are accordingly located by filtering the $n_{i, j}$ elements with value 4 . All the other matrix element are set to 0 , shown as black pixels.

It is possible to show that all the interesting relations of incidence/adjacency between cells of different dimensions can be both computed and efficiently queried by pairwise computing some matrix products, with one of terms possibly transposed, using only the two boundary and coboundary operator matrices $\left[\partial_{p}\right]$ and $\left[\delta_{p}\right]$, and where $\left[\delta_{p}\right]=\left[\partial_{p}^{\top}\right]$. We may also show that such matrices are very sparse, with their sparseness growing rapidly with their size (see Section 3.2). The pattern of non-zeros in matrix $\left[\partial_{3}\right]$, corresponding to a small brick of shape $(4,4,4)$, is given in Fig. 1.

\subsection{Multi-indices from Cartesian Indices}

In order to utilize the topological algebra shortly recalled in this paper, we need to explicitly sort the cells of the various dimensions into linearly ordered sequences, possibly according to the order in which their information is linearly accommodated in computer storage. In Julia, our algorithms are most efficiently written in terms 
of a single linear index $A[i]$, even if A is multi-dimensional. Regardless of the array's native indices, linear indices always range from $1:$ length $(A)$.

\subsection{Taubin Smoothing}

Brick joining Every boundary chain extracted from an image block $\mathbb{B}(i, j, k, n)$ is a 2-cycle, i.e., a closed 2chain, defined as a 2-chain with empty boundary. Such 2-cycles are joined together by removing the boundary artifacts, i.e., the double 2-cells at the boundaries of adjacent bricks, after having suitably shifted their indices to an unique linear representation of the whole surface. The resulting raster surface is composed of mutually orthogonal raster facets, and must be smoothed in order to get a fair surface.

Surface Smoothing A linear time and space algorithm for this purpose is the Laplacian smoothing, which iteratively moves each vertex ( 0 -cell) to the centroid of its neighbors. A well known weakness of this simple algorithm is the asymptotic convergence of the whole mesh to a single point, resulting in unfair size reduction even after few iterations. The Taubin smoothing algorithm [26, 27] alternates two Laplacian smoothing steps with shrink and inflate effects respectively, with the result of delivering pretty invariant size and volume of the smoothed mesh. The best results are obtained on meshes which have small variations of edge length and face angles, which is the case of surfaces extracted from 3D raster images.

\section{Brick-parametric Design}

In this section we discuss how the input 3D image is handled in order to exploit a data parallelism at brick-level. Specifically, we deal with the image decomposition via the brick operator, the brick-to-boundary mapping, and the embedding of the extracted (boundary) 2-cycles from local to global coordinates, in order to merge togheter the various surface patches.

\subsection{Brick Decomposition}

Let us assume that medical devices produce 3D images with lateral dimensions that are integer multiples of some power of two, like $128,256,512$, etc. Therefore, any cuboidal portion of the image is completely determined by the Cartesian indices of its voxels of lowest and highest indices, and is extracted by multidimensional array slicing as $\operatorname{image}\left(\ell_{x}: h_{x}, \ell_{y}: h_{y}, \ell_{z}: h_{z}\right)$.

For the sake of simplicity, we assume a common size on the three image axes, and the block image $\mathbb{B}$, called brick, as function of the element of lowest coordinates $i, j, k \in[0: n-1]$ and of block lateral size $n \in \mathbb{N}$ :

$$
\mathbb{B}(i, j, k, n):=\operatorname{image}(i: i+n, j: j+n, k: k+n)
$$

Figure 2a shows the brick decomposition in a $2 \mathrm{D}$ image, with positive integers $(u, v)$ giving the lateral sizes of the image. Note that brick sides do not necessarily correspond to image edges.

\subsection{Brick Operator}

Chain coordinates We are going to treat each image brick independently of each other. Hence, we map each image brick $\mathbb{B}(i, j, k, n)$ to the linear chain space $C_{3}$ of algebraic dimension $n \times n \times n$, using coordinate vectors $[c] \in\{0,1\}^{n^{3}}$, where each voxel (basis element $c \in C_{3}$ ) is mapped via Cartesian-to-linear map to a vector with $n^{3}-1$ zeros and only one element 1 . In other words, each voxel in a brick image will be seen as a basis binary vector, and (more interestingly) every subset of brick elements as the corresponding binary vector (sum of included basis vectors), with as many as the cardinality of the considered subset. 


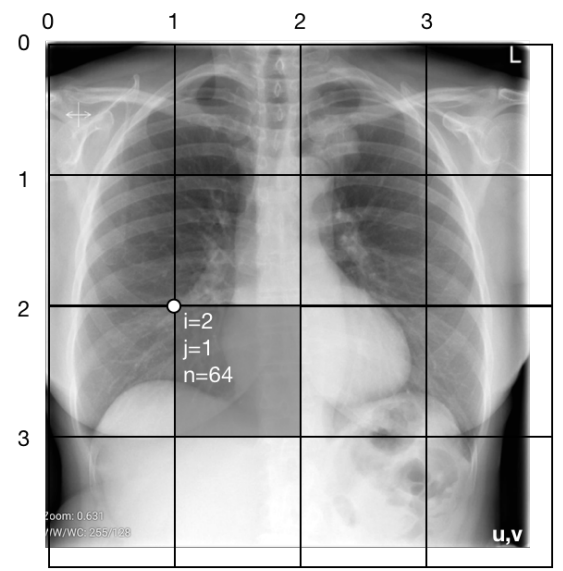

(a) A possible brick partitioning of a radiologic image. The evidenced 2D brick, of size $n^{d}=64^{2}$, is sliced by $\mathbb{B}([2,1,64])=\operatorname{Image}(128: 172,64: 128)$

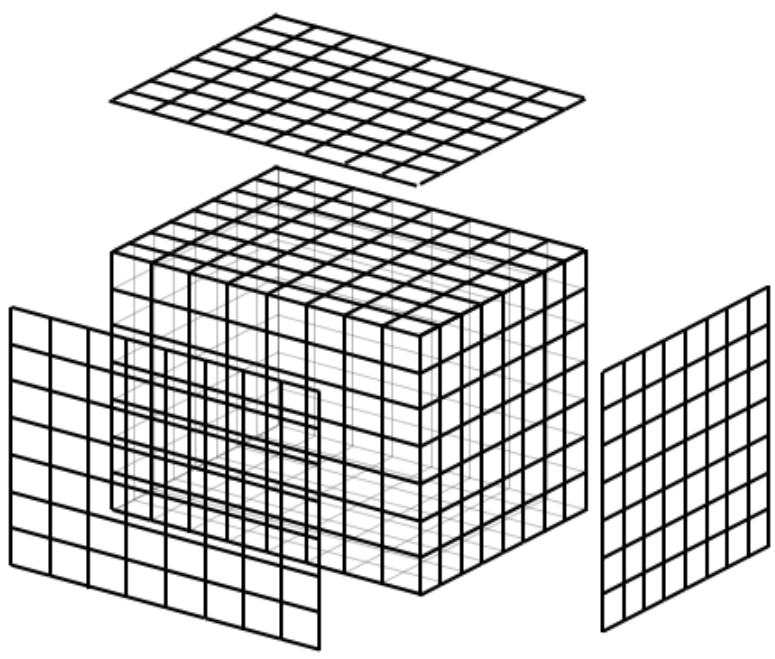

(b) Faces on the brick boundary

Figure 2: Brick decomposition

Boundary operator For a fixed brick size $n$, the boundary operator $\partial_{d}: C_{d} \rightarrow C_{d-1}$, with $d \in\{2,3\}$, will be constructed once and for all using the algorithm given in [17]. The corresponding computer code is inlined within the boundary extraction software.

It is easy to see that all matrices $\left[\partial_{d}\right]$ are very sparse, since they contains $2 d$ non-zeros (ones) for each column (of length $n^{d}$ ), i.e., 4 ones or 6 ones per columns for the 2D and 3D case, respectively. We remind that the matrix of a linear operator between linear spaces contains by columns the basis element of the domain, represented in the target space. In our case, the former is an image element (2-cube or 3-cube), represented as the chain of its boundary cells, i.e. either a 1-cycle of 4 edges (2D), or a 2-cycle of 6 faces (3D), respectively.

The number of rows of $\left[\partial_{d}\right]$ equals the dimension of the linear space $C_{d-1}$, i.e., the number of $(d-1)$-cells of the cellular partition of the image. We compute their number in two steps: (a) first, we map one-to-one the $n^{d} d$-cells with $d$ adjacent $(d-1)$-cells, so getting $d n^{d}$ distinct basis elements of $C_{d-1}$; (b) then, we complete the basis by adjoining $n^{d-1}$ boundary elements for each of the $d$ dimensions of the brick, so providing further $d n^{d-1}$ basis elements for $C_{d-1}$. The dimension of $C_{d-1}$, and therefore the number of rows of $\left[\partial_{d}\right]$ matrix is $d\left(n^{d-1}+n^{d}\right)=d n^{d-1}(1+n)$. The number of columns of $\left[\partial_{d}\right]$ equals the number of basis elements of $C_{d}$, i.e., the number $n^{d}$ of brick elements.

Sparsity and size of the boundary matrix As we have seen, we have $2 d$ non-zero elements for each column of $\left[\partial_{d}\right]$, so that the total number of non-zeros is $2 d n^{d}$. The number of matrix element is given by the number of rows $\left((d-1)\right.$-cells), times the number of elements per row, i.e., $d n^{d-1}(1+n) \times n^{d}$, giving $0 \leq$ sparsity $\leq 1$, with:

$$
\text { sparsity }=1-\frac{\text { non-zero elements }}{\text { total elements }}=1-\frac{2 d \times n^{d}}{d n^{d-1}(1+n) \times n^{d}}=1-\frac{2}{n^{d-1}+n^{d}}
$$

Using sparse matrices in CSC (Compressed Sparse Column) format we get a storage size

$$
\operatorname{space}\left(\partial_{d}\right)=2 \times \# \text { nzero }+\# \text { columns }=2 \times 2 d n^{d}+n^{d}=(4 d+1) n^{d} .
$$




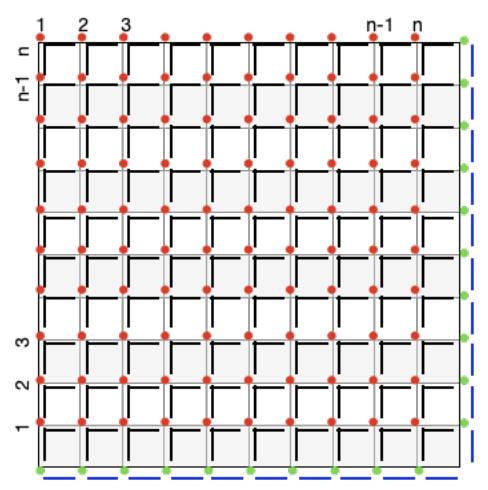

Figure 3: Mapping between 2-cells and 1-cells, used to compute the size of [ $\left.\partial_{2}\right]: C_{2} \rightarrow C_{1}$ for a 2D brick of size $n^{d}=10^{2}$. The dimensions of the chain spaces are: $\operatorname{dim} C_{2}=n^{d}=100, \operatorname{dim} C_{1}=d n^{d}+d n^{d-1}=200+20$.

In conclusion, for brick size $n=64$, the matrix $\left[\partial_{d}\right]$ requires for $2 \mathrm{D}$ bricks $9 \times 64^{2}=36,864$ memory elements, and for 3D bricks $13 \times 64^{3}=3,407,872$ memory elements. Counting the bytes for the standard implementation of a sparse binary matrix ( 1 byte for values and 8 bytes for indices) we get $(18 d+8) n^{d}$ bytes, so requiring $180 \mathrm{~KB}$ for $2 \mathrm{D}$ brick and $12 \mathrm{MB}$ for $3 \mathrm{D}$ brick.

\subsection{Brick-to-boundary Mapping}

Here we refer directly to the 3D case. Let us call segment $S$ the bulk content of interest within the input 3D image of size $(u, v, w)$. Our aim is to compute the segment boundary $\partial_{3} S$. First we set the size $n$ of the brick, in order to decompose the input $i \operatorname{mage}(u, v, w)$ into a fair number $M$ of bricks:

$$
M=\lceil u / n\rceil \times\lceil v / n\rceil \times\lceil w / n\rceil \simeq \frac{u v w}{n^{3}} .
$$

Then, we consider each segment portion $c_{i, j, k}=S \cap \mathbb{B}(i, j, k, n)$ and compute its coordinate vector local to the brick $[c] \in C_{3}\left(n^{3}\right)$. This one is a sparse binary vector of length $n^{3}$. Then, we assemble by columns the $M$ representations $[c]_{j}(1 \leq j \leq M)$ of segment portions into a sparse binary matrix $\mathbf{S}$, of dimension $n^{d} \times M$, with $d=3$. Finally, we compute a matrix $\mathbf{B}$ of boundary portions of $S$, represented by columns as chain coordinate vectors in $C_{2}$ :

$$
\mathbf{B}=\left[\partial_{3}(n)\right] \mathbf{S},
$$

where the boundary matrix has dimension $d n^{d-1}(1+n) \times n^{d}$. Of course, the $\mathbf{B}$ sparse matrix has the same column number $M$ of $\mathbf{S}$, because each column contains the boundary representation of the corresponding $S \cap \mathbb{B}(i, j, k, n)$, and the number of matrix rows is the dimension $d n^{d-1}(1+n)$ of the linear space $C_{2}$.

Embedding Two final computational steps are required to embed the 2-chains in $\mathbb{E}^{3}$ space, and to assemble the whole resulting surface. In particular, we need to compute the embedding function $\mu: C_{0} \rightarrow \mathbb{E}^{3}$, where $C_{0}$ is the space of 0 -chains, one-to-one with the vertices of the extracted surface. The simplest solution is to associate four vertices to each 2-cell of the extracted surface, i.e., to each non-zero entry in every column of $\mathbf{B}$. The $\mu$ function can be computed by identifying, via element position in the column, a triple of integer values $0 \leq x \leq u, 0 \leq y \leq v$, and $0 \leq z \leq w$ for each vertex of the 2 -cell. The mapping can be implemented using a dictionary, that will store the inverse coordinate transformation used at the beginning, i.e., the one from Cartesian to linear coordinates, in order not to duplicate the output vertices. 


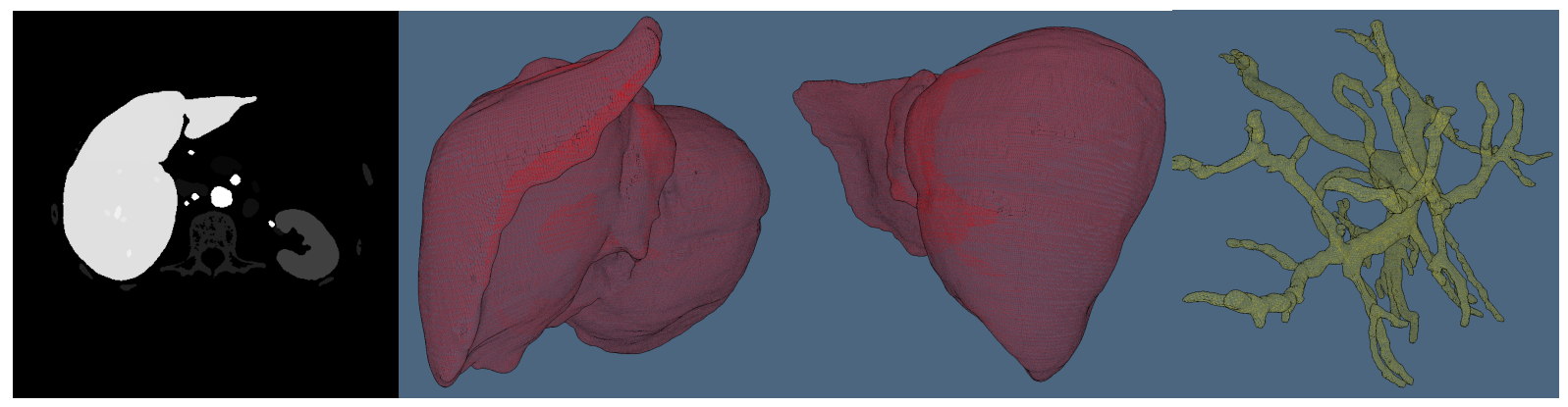

Figure 4: The left image shows one slice with segmented organs from the Ircad dataset [24]. The other three images show the surface of the liver and portal vein generated by the lar-surf.jl package.

\subsection{Brick-level Parallelism}

In the computational pipeline discussed in this paper, several steps can be efficiently performed in parallel at the image-brick level, due to the embarrassingly data-parallel nature of the problem. In particular, little effort is needed to split the problem into several parallel tasks $S_{i, j, k}$, using multiarray slicing. The granularity of parallelism, depending on the brick size $n$, is further exploited by the computation of a single boundary matrix $\left[\partial_{d}(n)\right]$, function only of brick size $n$, so that the initial communication cost of broadcasting the matrix to compute nodes can be carefully controlled, and finely tuned depending on the system architecture. The whole approach is appropriate for SIMD (Single Instruction, Multiple Data) hybrid architectures of CPUs and GPUs, since only the initial brick setup of the boundary matrix and image slices, as well as the final collection of computed surface portions, require inter-process communication.

\section{Julia Implementation}

The computer code is implemented in Julia language [3] according to the workflow described below, whose stages are parallelized and/or optimized in various ways. The workflow scheme can be seen in Fig. 5. The implementation is available on GitHub [11] and our LarSurf.jl package can be installed using a standard Julia package register.

\subsection{Parallel Workflow}

Workflow setup The functions in this preliminary step include:

1. input of $3 \mathrm{D}$ medical image $\mathcal{I}$ with shape $\left(\ell_{1}, \ell_{2}, \ell_{3}\right)$, such that: $\mathcal{I}=\left[\ell_{1}\right] \times\left[\ell_{2}\right] \times\left[\ell_{3}\right]$, where $\left[\ell_{k}\right]=$ $\left[1,2, \ldots, \ell_{k}\right]$

2. analysis of resources available in the computational environment, including operating system, type and number of compute nodes (processors, cores, GPUs), number of cores per node, RAM and cache amounts;

3. depending on the above, best choice of the size of $3 \mathrm{D}$ image brick $\mathcal{B}$. With default size $=64$, the number of bricks will be $n=\left\lceil\ell_{1} /\right.$ size $\rceil \times\left\lceil\ell_{2} /\right.$ size $\rceil \times\left\lceil\ell_{3} /\right.$ size $\rceil$. Hence the default number of bricks, of size $64^{3}$, is $m=8 \times 8 \times 4=256$, for standard medical images $512 \times 512 \times 256$;

4. computation of sparse brick boundary matrix $\left[\partial_{B}\right]$, where Int8 and Int64 are the types for values and indices, returning a sparse matrix value of type SparseMatrixCSC $\{$ Int8 $\}\{$ Int64\}, stored by Compressed Sparse Column $(\mathrm{CSC})$ format. The storage of $\left[\partial_{B}\right]$ (for $n=64$ ) requires about $12 \mathrm{MB}$; 
setup

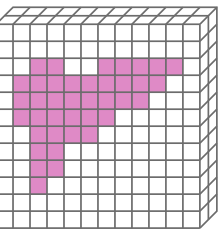

上ᄂ

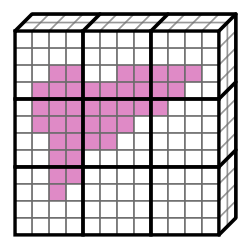

job enqueuing

multiplication coding
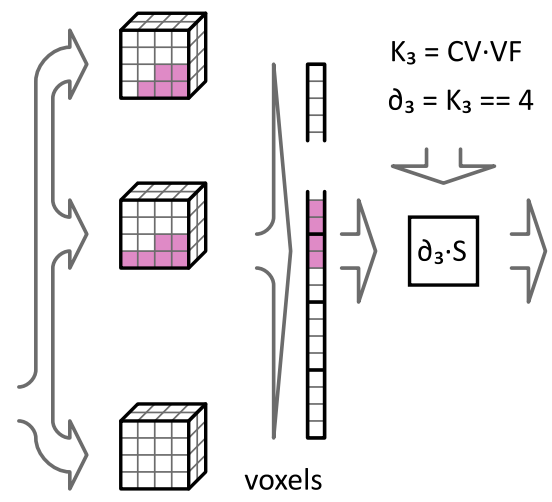

2-chain

decoding

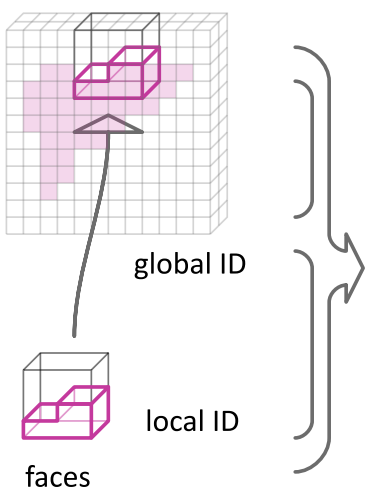

surface

assembling

Figure 5: Workflow of LAR-SURF algorithm

5. creation of either a local or distributed channel to implement a producer/consumer model of parallel/distributed computation, depending on available resources;

6. distribution of matrix $\left[\partial_{B}\right]$, of default size $12 \mathrm{MB}$, to all available nodes/cores (Julia workers), using the Julia macro @eveywhere.

Job enqueuing Communication and data synchronization are managed through Channels, which are the FIFO conduits that may provide producer/consumer communication. Overall execution time can be improved if other tasks can be run while a task is being executed, or while waiting for an external service/function to complete. The single work items of this stage follow:

1. extraction, from image arrays of the block views, depending on 3 Cartesian indices;

2. transform each block from global $\left[\ell_{1}\right] \times\left[\ell_{2}\right] \times\left[\ell_{3}\right]$ to local coordinates $[n] \times[n] \times[n]$;

3. further transform of each foreground voxel $\nu \in \mathcal{S} \subseteq \mathcal{I}$ from Cartesian to linear coordinates, using suitable functions from Julia's library.

4. enqueuing the job (as a sequence of integer positions for the non-zero image elements aligned in a memory buffer of proper Channel type).

3-Chain encoding The interesting part of the Image $\mathcal{I}$ is called Segment $\mathcal{S}$. The goal of the whole workflow is to extract a boundary model of $\mathcal{S}$ from $\mathcal{I}$. The portion of $\mathcal{S}$ inside $\mathcal{B}$, will be denoted as $\mathcal{S}(B)$. Each block $\mathcal{B}$ of the $3 \mathrm{D}$ image must by converted into the coordinate representation of a vector $\nu \in C_{3}$ in the linear space of 3-chains.

In coordinates local to $\mathcal{B}$, once an ordering from Cartesian to linear coordinates has been fixed, this vector is represented by a binary array of length size $e^{3}$. With size $=64$, we have $64^{3}=262144$, with a non-zero 
value (i.e. 1) for each foreground voxel in $\mathcal{S}(B)$. Therefore, the coded segment portion $\mathcal{S}(B)$ results in a space occupancy of about $262 \mathrm{~KB}$ if encoded as a full array (i.e., including zero values). When encoded as a sparse vector, its space occupancy will correspondingly decrease.

1. each encoding task produces either a full or sparse binary vector. We get either $262 \mathrm{~KB}$ or less per job, depending on the use of either a full vector, or a sparse one;

2. a special format for sparse CSC (Compressed Sparse Column) vectors can be used, since the value data for non-zeros does not need storage. Hence only a single 1-array of Int64 row positions (with total length equal to the number of non-zeros in the block, with $8 \times \mathrm{nnz} \mathrm{kB}$ storage) is needed;

3. prepare sequences of such data vectors, in order to feed efficiently the available processor threads.

4. In case of presence of one/more GPUs, a smaller size of the block is preferable for speed, even with smaller boundary matrices and higher numbers of coded vector chains.

SpMM Multiplication According to current literature [4], it is more convenient to execute SpMV (sparse matrix-vector) multiplications than SpMSpV (sparse matrix-sparse vector) multiplications. Since we have 256 such jobs (one multiplication per block) to perform in the default setting of the algorithm ( size of the block $64^{3}$; the size of the image $512^{2} \times 256$ ), or more in case of either smaller blocks or image larger than the standard one, this stage must be evidently parallelized and carefully tuned, possibly by using the GPU, if available.

1. the total speed of this stage is strongly dependent on the hardware available, on the granularity of bricks, and on the choice between dense/sparse storage of encoded 3-chains;

2. the compute elements or threads is fed without solution of continuity in a dataflow process. This parallel operation is, according to our preliminary experiments, the critical one of the whole workflow, since any $\Delta T$ (either positive or negative) in this stage contributes to the total time $T$.

2-Chain decoding Each multiplication of $\left[\partial_{B}\right]: C_{3} \rightarrow C_{2}$, times a 3-chain $\nu \in C_{3}$, produces a 2-chain $\sigma \in C_{2}$, i.e., the coordinate representation of the boundary vector $\sigma \in C_{2}$. The inverse of the coding algorithm is executed in the present stage. This process can also be partially superimposed in time with the previous ones, depending on the size of the memory buffers used to feed the CPU cores or the GPUs and get their results. Its elementary steps are as follows:

1. reading of position of ones (non-zeros) in the 2-chain as linear indices of rows;

2. conversion from linear indices to Cartesian indices in coordinates local to the $\mathcal{B}$ block, using the appropriate library functions;

3. conversion from each Cartesian index value to a suitably oriented (i.e., with proper attitude) geometry quadrilateral (or pair of triangles) in local coordinates.

Julia's vectorized pipeline data-flow was the more appropriate model to feed the workers' jobs. 
Assembling and artifact filtering The results of the previous stages can be described as a collection of sets of geometric quadrilaterals (quads), each one encoded as an array of quadruples of integer indices, pointing to the linear array of grid vertices associated with the image block $\mathcal{B}$. In other words, all quads of each job are now given in the same local coordinates. Besides putting each partial surface $\mathcal{S}(B)=\left(\mathrm{V}_{B}, \mathrm{FV}_{\sigma}\right)$ in the global coordinate system of the image, the present stage must eliminate the redundant boundary features possibly generated at the edges of the partial surface $\mathcal{S}(B)$ within each block $\mathcal{B}$ :

1. translate each array $\mathrm{FV}_{\sigma}$, of type Lar. Cells, by summing each vertex index to the linearized offset of the Cartesian coordinates $(i, j, k)$ of the $\mathcal{B}$ 's reference vertex, i.e., the one with lowest Cartesian coordinates within the $\mathcal{B}$ block.

2. remove both instances of double quads generated by Lar software at the block boundaries (see Fig. 2b). These are artifacts generated by the decomposition of the whole image into a number of blocks of tractable size.

3. a smart strategy to remove such artifacts was used, that does not require any sorting nor searching on the assembled array of quads. The output set of 2-cells (or triangles) is just rewritten, discarding the elements pointing to all vertices with one coordinate equal to a block boundary. A proper software filter was applied to this purpose.

Smoothing The final smoothing of the generated surfaces cannot be performed block-wise since this would introduce non-smooth artifacts at the block boundaries. Anyway, Taubin smoothing [25] can be performed in parallel, since for each vertex in the final surface (except eventually the ones on the image $\mathcal{I}$ boundaries) it essentially consists in computing a new position as a proper average of its neighboring vertices, i.e., by applying a discrete Laplacian operator. Some appropriate sets of workers may so be assigned the task of generating iteratively a new position for the vertices they take cure of. In particular, we have:

1. job enqueuing, by writing sets of integers (global linear indices of vertices) in array buffers of type Channel;

2. vectorized computation of proper averages of near vertices;

3. job dequeuing, by recovering finished tasks from a channel and assembling the results into the embedding function $\mathrm{V}: C_{0} \rightarrow \mathbb{E}^{3}$, providing an array of type Lar. Points of Float64 $\times 3$, with vertex coordinates by column.

\subsection{Performance Analysis}

Boundary matrix size The size of the boundary matrix is a critical parameter of the LAR-SURF method. To determine the optimal size of the boundary matrix we experimented on artificial data (Fig. 6a). The size of the experimental data was set to $512 \times 512 \times 512$ (a typical size of Computed Tomography medical images). Computation was done on the Tesla DGX-1 machine.

According to the experiment the fastest computation is with boundary matrix size $64 \times 64 \times 64$. This is an expected result. The larger boundary matrix is too big to fit in CPU's cache memory.

Comparison with the Marching Cubes algorithm To compare the time requirements of LAR-SURF with Marching Cubes implemented in Python we performed an experiment on Ircadb dataset [24]. Dataset contain 20 Computed Tomography images (see table 1) with xy-resolution from $0.56 \mathrm{~mm}$ to $0.87 \mathrm{~mm}$ and z-resolution from $1.0 \mathrm{~mm}$ to $4.0 \mathrm{~mm}$. The number of slices is each series varies from 74 to 260 and the size of each slice is $512 \times 512$. The dataset contains manually segmented liver, portal vein, and other structures. We performed 


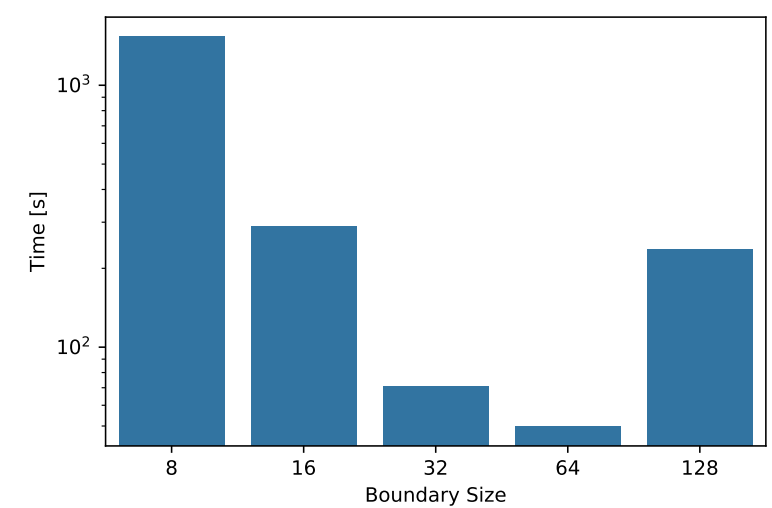

(a) Time requirements of the LAR-SURF filter used on artificial volumetric data with various sizes of boundary matrix

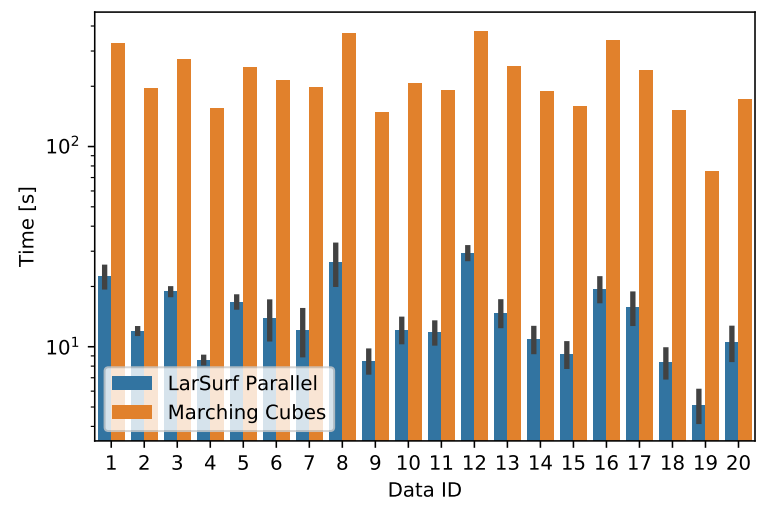

(b) Time requirements of the LAR-SURF filter and Marching Cubes on the Ircad dataset. Error bars show the 95\% confidence interval

Figure 6: Performance analysis of the LAR-SURF filter.

surface extraction of the liver with Marching Cubes and LAR-SURF. The time required for computation can be seen in Fig. $6 \mathrm{~b}$.

Based on the t-test with $\alpha=0.99, p=8.735 \times 10^{-24}$ and $s=-16.67$ it can be shown that the mean time consumed by LAR-SURF is significantly smaller than that consumed by Marching Cubes.

\section{Examples}

Use of the LarSurf.jl package can be seen on listings 1 where the liver segmentation with 2865131 voxels from the Ircad dataset is used as an input for our surface extraction algorithm. The size of 3D volumetric image is $129 \times 512 \times 512$ and the voxel resolution is $1.6 \times 0.57 \times 0.57[\mathrm{~mm}]$. The output liver surface model is formed by 182124 triangles and the number of vertices is 90822 . The visualization can be seen in Fig. 7 .

\section{Listing 1: Get surface from DICOM volumetric data}

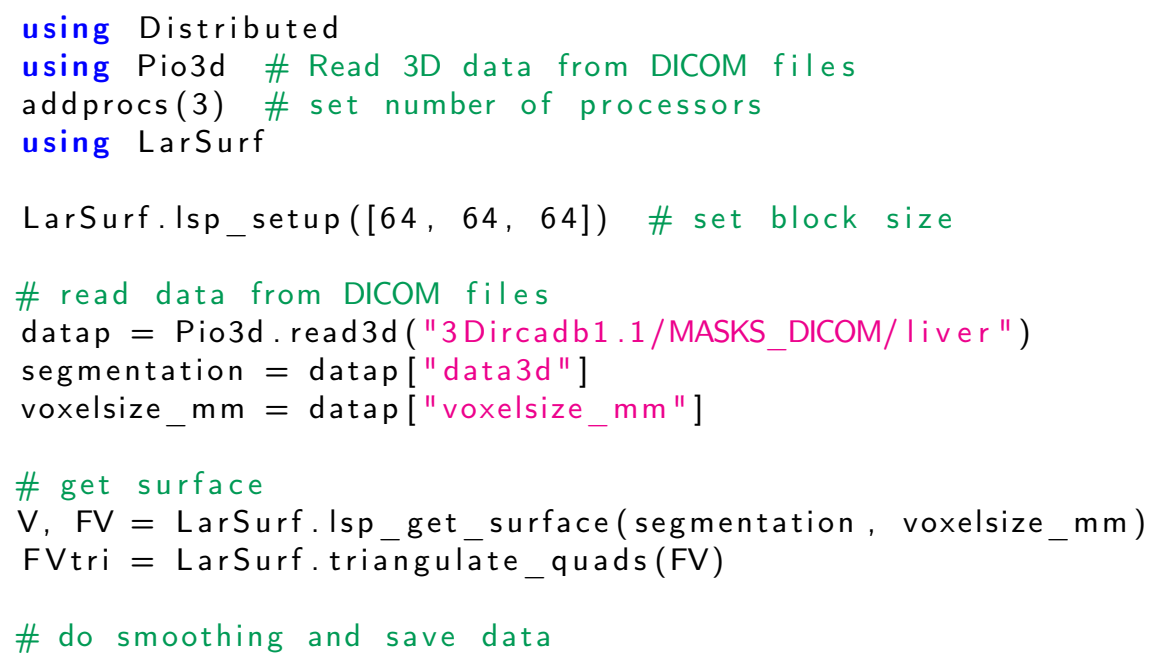



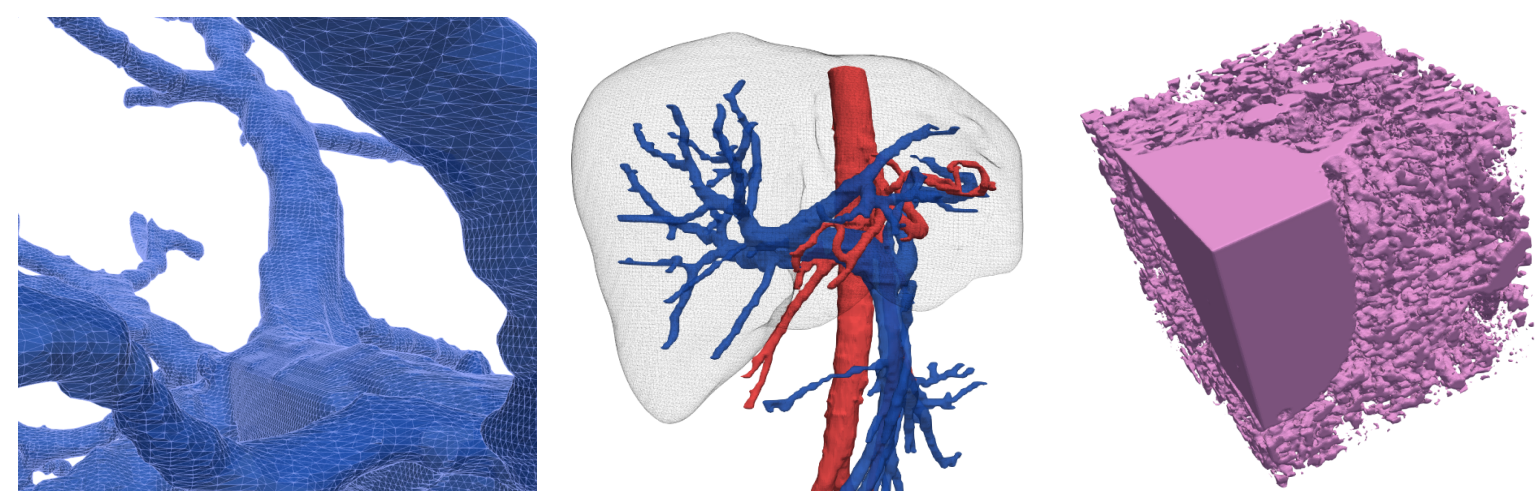

Figure 7: Triangulated surfaces of the macroscopic and microscopic structures of the liver extracted by the LAR-SURF algorithm. (a) Detail of the portal vein (PV) with resolution $1.6 \times 0.57 \times 0.57[\mathrm{~mm}]$. (b) model of the human liver, with portal vein and hepatic artery connected to colon and stomach. (c) microvasculature of a pig liver, based on corrosion cast of interlobular veins prepared by Eberlova [10]. The size of the specimen is $0.936[\mathrm{~mm}]$ along each axis and the resolution of the Micro-CT data is $4.682 \mu \mathrm{m}$. Note that brick boundaries are flat.

$V_{s}=$ LarSurf. Smoothing. smoothing_FV_taubin (V, FV, $\left.0.5,-0.2,40\right)$

objlines = LarSurf.Lar.Iar2obj( $\overline{\text { Vs }}, \overline{F V t r i, ~ " l i v e r . o b j ") ~}$

The portal vein surface extraction can be performed with a small change of the input path in the code. The 3D image resolution is the same. The number of input voxels is 103533 . The output surface is created by 90822 vertices and 182124 triangles.

The right image of Fig. 7 is the surface of the microvasculature of a pig liver. The volumetric image is based on Micro-CT data of corrosion casts of pig liver. [10]. The size of the visualized data is $100 \times 100 \times 100$ voxels and the size of the voxel is $4.682 \mu \mathrm{m}$. The number of triangles is 544784 and the number of vertices is 272826 .

\section{Discussion of Method}

Most other methods for extraction of boundary surfaces from 3D data arrays—including [28] and [29]—use implicit functions, defined by first averaging upon 3D mesh vertices the lighting or brightness of incident voxels, and then by applying some marching cube algorithm in order to traverse and to triangulate the iso-valued boundary patches.

We use instead, we use a binary labeling of the voxel sets of the image segment of interest, and the standard medical 3D image array as solid representation. The set of boundary facets is extracted through spMV (sparse matrix vector multiplication) of the $\left[\partial_{3}\right]$ matrix times the binary vector labeling the voxels of the segment. The boundary matrix is computed once and for all, sent to all workers (cores or nodes), then used in parallel for all image brick extractions. This algebraic method can be immediately extended to multi-material processing, as well as to designing multi-material tissue layering for 3D printing.

In particular, we might organize a multi-material (or multi-organ) extraction, simply by multiplication of the boundary matrix $\left[\partial_{3}\right]: C_{3} \rightarrow C_{2}$ times a binary matrix where the non-zero elements on each column represent either one of the materials, or one of the organs to be algebraically extracted by the filter. The two surface patches common to two adjacent segments will be exactly coincident, and share the same geometry and topology, with the only exception of the each patch contours, where the influence of adjacent patches induces each instance to be separated and rounded-off.

The main advantage of the approach discussed in this paper is given by its very algebraic nature; our 
implementation does not need any kind of algorithmic graph traversal, of difficult parallelization, but only the execution of standard algebraic computing kernels, and in particular the spMV and the spMspM (sparse matrix-sparse matrix multiplication) kernels, nowadays efficiently implemented by tensor processors on Nvidia hardware. As we have shown, the parallelization, both shared-memory and distributed, is also fairly easy and with good speed-up.

\section{Conclusion}

We introduced a Julia implementation of an algebraic filter to extract the boundary surface of some specific image segment, described as a 3-chain of voxels from 3D medical images. Translation from Cartesian indices of cells to linearized indices, computation of sparse boundary matrices, and sparse matrix-vector multiplication are the main computational kernels of this approach.

The implementation of the LAR-SURF filter is available in an open-source repository and can be installed using standard Julia package manager [11].

We showed a good speed-up over marching-cubes algorithms. The existing implementation employs Julia's channels for multiprocessing. Our performance experiment determined an optimal size of the brick size. Parallelization makes a large portion of spared computational cost. Moreover, we expect additional improvements in the future because our approach is appropriate for SIMD (Single Instruction, Multiple Data) hybrid architectures of CPUs and GPUs, since only the initial block setup of the boundary matrix and image slices and the final collection of computed surface portions require inter-process communication.

Currently, the computational pipeline is being strongly improved to gain a greater speed-up using native Julia implementation CUDA.jl of Nvidia programming platform [2] and Julia's SuiteSparseGraphBLAS.j1 framework [4] for graph algorithms with the language of linear algebra. In particular, we are extending the use-pattern of this library, in order to work with general cellular complexes.

\section{ACKNOWLEDGEMENTS}

This work was supported by Charles University Research Centre program UNCE/MED/006 "University Center of Clinical and Experimental Liver Surgery" and Ministry of Education project ITI CZ.02.1.01/0.0/0.0/17_048/0007280: Application of modern technologies in medicine and industry. The research was also supported by the project LO 1506 of the Czech Ministry of Education, Youth and Sports.

\section{A Appendix}

Here we provide, for the sake of readers, a list of symbols used in the paper, and a small set of related definitions. A description of public dataset used in the experiments, and a coding example of computation of the sparse $\left[\partial_{3}\right]$ boundary matrix in Julia is finally given.

\subsection{Symbol list}

$\mathcal{I}$ three-dimensional medical image

$\ell_{1}, \ell_{2}, \ell_{3}$ dimensions of image

$\mathcal{S}$ segment: a subset of voxels from image segmentation

$\mathcal{B}$ 3D image brick

size lateral dimension of cubic brick $\mathcal{B}$

$n$ number of bricks $\mathcal{B}$ (jobs) in $\mathcal{I}$

$\left[\partial_{\mathcal{B}}\right]$ boundary matrix for brick $\mathcal{B}$

$C_{p}$ linear (vector) space of $p$-chains

$\nu \in C_{p}$ p-chain 
[ $\nu$ ] coordinate representation (binary vector) of $\nu$

$\mathbb{E}^{3}$ Euclidean 3-space

\subsection{Definitions}

Boundary model Closed manifold surface of the boundary of a solid model

CSC Compressed Sparse Column format for sparse matrices

Global coordinates Integer linear coordinates of $\mathcal{I}$

Local coordinates Integer linear coordinates of $\mathcal{B}$

Cartesian coordinates Integer triples $(i, j, k)$ one-to-one with voxels

Voxels Individual elements in 3D image (3-cells)

$p$-chain Formal linear combination of $p$-cells with coefficients in $\{0,1\}$

Coord. repr. Binary vector (for $p$-chains) or binary matrix (for chain operators)

Quad Geometric quadrilateral; convex polygon with four vertices

Foreground voxel Individual element of a segment $\mathcal{S}$

Segment Subset of voxels resulting from image segmentation

\subsection{D-Ircadb Dataset}

For perfomance analysis the public dataset from the Research Institute against Digestive Cancer (IRCAD) [24] was used. Table 1 describes the dataset.

\begin{tabular}{lrrrrr}
\hline & z-resolution [mm] & xy-resolution $[\mathrm{mm}]$ & obj. voxels & size xy & size z \\
\hline $\min$ & 1.00000 & 0.561000 & $5.832080 \mathrm{e}+05$ & 512.0 & 74.000000 \\
mean & 1.77750 & 0.725141 & $1.894777 \mathrm{e}+06$ & 512.0 & 141.150000 \\
$50 \%$ & 1.60000 & 0.739094 & $1.760604 \mathrm{e}+06$ & 512.0 & 127.000000 \\
$\max$ & 4.00000 & 0.873047 & $3.341433 \mathrm{e}+06$ & 512.0 & 260.000000 \\
\hline
\end{tabular}

Table 1: Ircad dataset description [24]. It contains 20 Computed Tomography images of the abdomen with manually segmented tissues.

\subsection{Basic operations in LAR}

Boundary matrices for grids of cubes:

We give here the full Julia code for the algebraic computation of $\partial_{3}$ matrix, for a very small : grid of unit 3 -cubes. Due to the simplicity of the cells (voxels = cubes), a sufficient (geom,top) pair is given below as $(\mathrm{V}, \mathrm{CV})$, where $\mathrm{CV}$ is an array of arrays of Int64 indices of vertices of grid cubes.

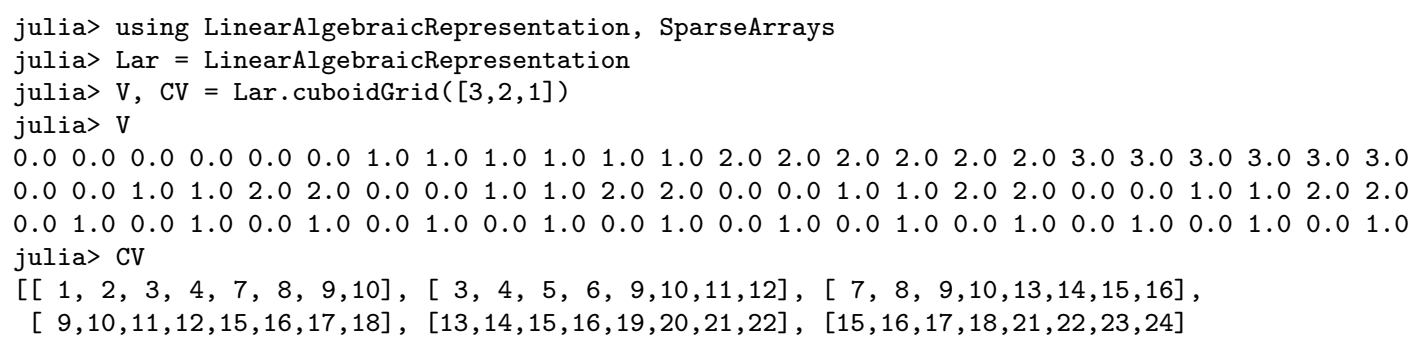


Face and Edge Data generation:

In the following, we provide the functions for generating the face data FV (vertex indices in faces) with function CV2FV and edge data EV (vertex indices in edges) with function CV2EV from cell data CV.

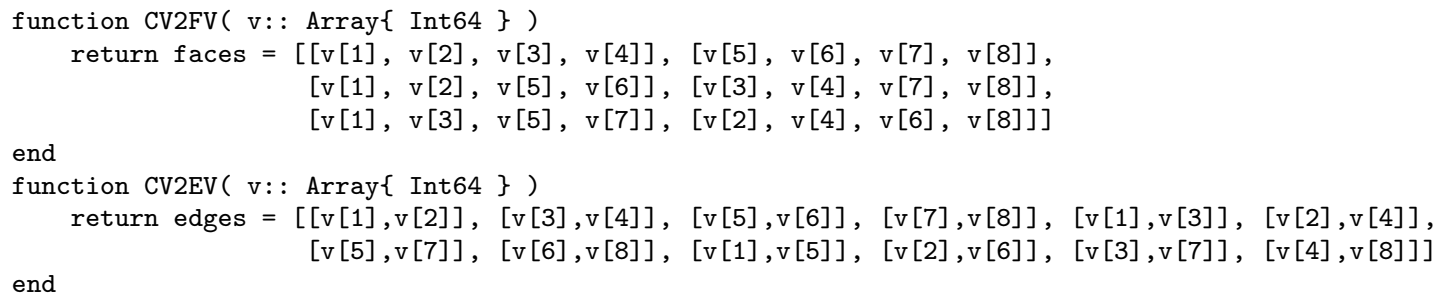

\section{Characteristic matrices:}

The function $\mathrm{K}$ transforms an array of arrays ( $\mathrm{VV}, \mathrm{EV}, \mathrm{FV}, \mathrm{CV}$ ) into a sparse binary characteristic matrix $\left(M_{0}, M_{1}, M_{2}, M_{3}\right)$. A Julia sparse matrix needs three arrays I, J, Vals of rows, columns, values of non-zeros:

$\mathrm{VV}=[\mathrm{v}]$ for $\mathrm{v}=1: \operatorname{size}(\mathrm{V}, 2)]$;

$\mathrm{FV}=\operatorname{collect}(\operatorname{Set}\{\operatorname{Array}\{\operatorname{Int} 64,1\}\}(\operatorname{vcat}(\operatorname{map}(\mathrm{CV} 2 \mathrm{FV}, \mathrm{CV}) \ldots)))$

$[[13,15,19,21],[1,2,3,4],[7,9,13,15],[13,14,15,16],[7,8,13,14],[1,2,7,8],[2,4,8,10],[7,8,9,10]$,

$[3,5,9,11],[8,10,14,16],[15,16,21,22],[9,11,15,17],[3,4,5,6],[17,18,23,24],[11,12,17,18]$,

$[1,3,7,9],[3,4,9,10],[9,10,15,16],[4,6,10,12],[13,14,19,20],[9,10,11,12],[15,16,17,18]$,

$[19,20,21,22],[15,17,21,23],[16,18,22,24],[21,22,23,24],[10,12,16,18],[5,6,11,12],[14,16,20,22]]$

$\mathrm{EV}=\operatorname{collect}(\operatorname{Set}\{\operatorname{Array}\{\operatorname{Int} 64,1\}\}(\operatorname{vcat}(\operatorname{map}(\mathrm{CV} 2 \mathrm{EV}, \mathrm{CV}) \ldots)))$

$[[15,17],[16,22],[6,12],[17,23],[18,24],[4,10],[3,4],[13,15],[11,12],[9,15],[13,19]$,

$[1,7],[5,11],[5,6],[12,18],[8,14],[15,21],[17,18],[1,3],[2,4],[16,18],[2,8],[21,23]$,

$[20,22],[1,2],[14,16],[10,16],[13,14],[19,21],[7,13],[9,10],[23,24],[11,17],[21,22]$,

$[3,9],[3,5],[9,11],[7,9],[14,20],[7,8],[22,24],[19,20],[8,10],[15,16],[10,12],[4,6]]$

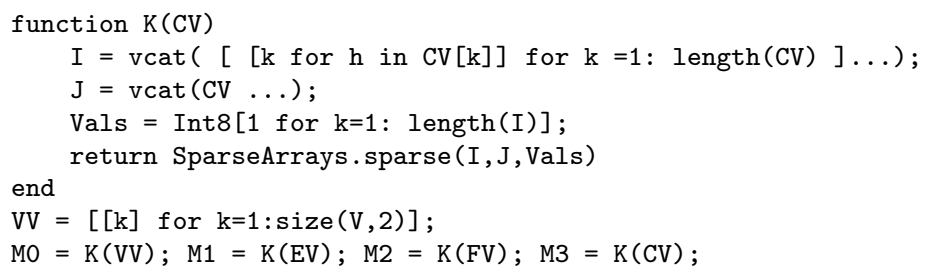

\section{Boundary matrices:}

The boundary matrices between non-oriented chain spaces are computed by sparse matrix multiplication followed by matrix filtering, produced in Julia by the broadcast of vectorized integer division $(. \div)$ :

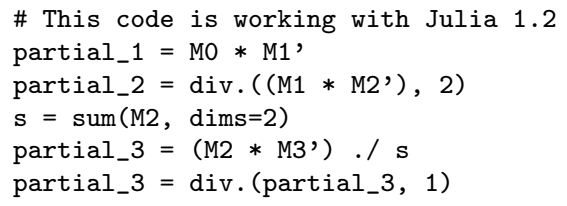

Miroslav Jirik (D) http://orcid.org/0000-0002-8002-2079

Antonio DiCarlo (D) http://orcid.org/0000-0002-7736-684X 
Václav Liska (D) http://orcid.org/0000-0002-5226-0280

Alberto Paoluzzi (D) http://orcid.org/0000-0002-3958-8089

\section{REFERENCES}

[1] Bajaj, C.L.; Pascucci, V.; Thompson, D.; Zhang, X.Y.: Parallel accelerated isocontouring for out-of-core visualization. Proceedings of the 1999 IEEE Symposium on Parallel Visualization and Graphics, PVGS 1999, 97-104, 1999. http://doi.org/10.1145/328712.319342.

[2] Besard, T.; Foket, C.; De Sutter, B.: Effective Extensible Programming: Unleashing Julia on GPUs. IEEE Transactions on Parallel and Distributed Systems, 30(4), 827-841, 2019. ISSN 15582183. http: //doi.org/10.1109/TPDS.2018.2872064.

[3] Bezanson, J.; Edelman, A.; Karpinski, S.; Shah, V.B.: Julia: A fresh approach to numerical computing. SIAM Review, 59(1), 65-98, 2017. http://doi.org/10.1137/141000671.

[4] Buluc, A.; Mattson, T.; McMillan, S.; Moreira, J.; Yang, C.: Design of the GraphBLAS API for C. In Proceedings - 2017 IEEE 31st International Parallel and Distributed Processing Symposium Workshops, IPDPSW 2017, 643-652, 2017. ISBN 9781538634080. http://doi.org/10.1109/IPDPSW.2017.117.

[5] Chernyaev, E.: Marching cubes 33: Construction of topologically correct isosurfaces. Tech. rep., 1995. http://wwwinfo.cern.ch/asdoc/psdir.

[6] Crossno, P.; Angel, E.: Isosurface extraction using particle systems. Proceedings of the IEEE Visualization Conference, 495-498, 1997. http://doi.org/10.1109/visual.1997.663930.

[7] Custodio, L.; Pesco, S.; Silva, C.: An extended triangulation to the Marching Cubes 33 algorithm. Journal of the Brazilian Computer Society, 25(1), 6, 2019. ISSN 1678-4804. http://doi.org/10. 1186/s13173-019-0086-6.

[8] Dicarlo, A.; Milicchio, F.; Paoluzzi, A.; Shapiro, V.: Chain-based representations for solid and physical modeling. IEEE Transactions on Automation Science and Engineering, 6(3), 454-467, 2009. ISSN 15455955. http://doi.org/10.1109/TASE. 2009. 2021342.

[9] Dicarlo, A.; Paoluzzi, A.; Shapiro, V.: Linear algebraic representation for topological structures. Comput. Aided Des., 46, 269-274, 2014. ISSN 0010-4485. http://doi.org/10.1016/j.cad.2013.08.044.

[10] Eberlova, L.; Liska, V.; Mirka, H.; Tonar, Z.; Haviar, S.; Svoboda, M.; Benes, J.; Palek, R.; Emingr, M.; Rosendorf, J.; others: The use of porcine corrosion casts for teaching human anatomy. Annals of Anatomy-Anatomischer Anzeiger, 213, 69-77, 2017.

[11] Jirik, M.; Paoluzzi, A.: LarSurf.jl package on GitHub, 2020. https://mjirik.github.io/LarSurf . jl/.

[12] Lachaud, J.O.; Montanvert, A.: Continuous analogs of digital boundaries: A topological approach to iso-surfaces. Graphical Models, 2000. ISSN 15240703. http://doi.org/10.1006/gmod.2000.0522.

[13] Lorensen, W.E.; Cline, H.E.: Marching Cubes: A High Resolution 3D Surface Construction Algorithm. ACM siggraph computer graphics, 21(4), 163-169, 1987.

[14] Natarajan, B.K.: On generating topologically consistent isosurfaces from uniform samples. The Visual Computer, 11(1), 52-62, 1994. ISSN 1432-2315. http://doi.org/10.1007/BF01900699.

[15] Newman, T.S.; Yi, H.: A survey of the marching cubes algorithm. Computers and Graphics (Pergamon), 30(5), 854-879, 2006. ISSN 00978493. http://doi.org/10.1016/j.cag.2006.07.021.

[16] Paoluzzi, A.; Dicarlo, A.; Furiani, F.; Jirik, M.: CAD models from medical images using LAR. ComputerAided Design, 13(6), 2016. ISSN 0010-4485. http://doi.org/10.1080/16864360.2016.1168216. 
[17] Paoluzzi, A.; Shapiro, V.; DiCarlo, A.; Furiani, F.; Martella, G.; Scorzelli, G.: Topological computing of arrangements with (co)chains. Transactions on Spatial Algorithms and Systems (ACM TSAS), 2020. Accepted for publication.

[18] Paoluzzi, A.; Shapiro, V.; DiCarlo, A.; Scorzelli, G.; Onofri, E.: Finite Boolean Algebras for Solid Geometry using Julia's Sparse Arrays. arXiv e-prints, arXiv:1910.11848, 2019. https://arxiv.org/pdf/1910. 11848.

[19] Paoluzzi, A.; Shapiro, V.; DiCarlo, A.; Scorzelli, G.; Onofri, E.: Finite boolean algebras for solid geometry using julia's sparse arrays, 2019. https://arxiv.org/abs/1910.11848. Eprint in https://arxiv.org/abs/1910.11848.

[20] Requicha, A.G.: Representations for rigid solids: Theory, methods, and systems. ACM Comput. Surv., 12(4), 437-464, 1980. ISSN 0360-0300. http://doi.org/10.1145/356827.356833.

[21] Rohan, E.; Lukes, V.; Jonásová, A.: Modeling of the contrast-enhanced perfusion test in liver based on the multi-compartment flow in porous media. Journal of Mathematical Biology, 77(2), 421-454, 2018. ISSN 14321416. http://doi.org/10.1007/s00285-018-1209-y.

[22] Shapiro, V.: Solid modeling. In G. Farin; J. Hoschek; S. Kim, eds., Handbook of Computer Aided Geometric Design, chap. 20, 473-518. Elsevier Science, 2002.

[23] Smistad, E.; Elster, A.C.; Lindseth, F.: Real-Time Surface Extraction and Visualization of Medical Images using OpenCL and GPUs. In Norsk informatikkonferanse, 141-152, 2012. http://www.tapironline. no/last-ned/1050.

[24] Soler, L.: 3D-IRCADb-01 dataset, 2016. https://www.ircad.fr/research/3dircadb/.

[25] Taubin, G.: Curve and surface smoothing without shrinkage, 1995. http://doi.org/10.1109/iccv. 1995.466848.

[26] Taubin, G.: A signal processing approach to fair surface design. In Proceedings of the 22Nd Annual Conference on Computer Graphics and Interactive Techniques, SIGGRAPH '95, 351-358. ACM, New York, NY, USA, 1995. ISBN 0-89791-701-4. http://doi.org/10.1145/218380.218473.

[27] Taubin, G.: Geometric Signal Processing on Polygonal Meshes. In Eurographics 2000 - STARs. Eurographics Association, 2000. ISSN 1017-4656. http://doi.org/10.2312/egst. 20001029.

[28] Wang, C.C.L.: Direct extraction of surface meshes from implicitly represented heterogeneous volumes. Comput. Aided Des., 39(1), 35?50, 2007. ISSN 0010-4485. http://doi.org/10.1016/j.cad. 2006. 09.003.

[29] Wang, C.C.L.: Extracting Manifold and Feature-Enhanced Mesh Surfaces From Binary Volumes. Journal of Computing and Information Science in Engineering, 8(3), 2008. ISSN 1530-9827. http://doi.org/ 10.1115/1.2960489. 031006. 